\title{
DNA-functionalized photonic crystal microspheres for multiplex detection of toxic metal ions
}

\author{
Zhengyu Yan, Chunqing Tian, Xincheng Qu, Weiyang Shen*, Baofen Ye* \\ Key Laboratory of Biomedical Functional Materials, School of Science, China Pharmaceutical University. Tongjia Xiang 24, Nanjing, Jiangsu, 210009, P.R.
}

A B S TR A C T

A novel type of suspension array for multiplex detection of heavy metal ions with photonic crystal hydrogel microspheres was reported. The photonic crystal hydrogel microspheres have close-packed photonic crystal particles as their encoding units and ssDNA-functionalized hydrogel as the sensing units. The developed microspheres were successfully applied in multiplex detection of heavy metal ions.

Keywords: photonic crystal; colloidal crystal; multiplex assay; aptamer; heavy metal ions

\section{Introduction}

With the increasing activities of modern industries, toxic heavy metal pollution has become a critical worldwide issue[1]. Heavy metal ions such as mercury $\left(\mathrm{Hg}^{2+}\right)$ and silver $\left(\mathrm{Ag}^{+}\right)$are severe pollutants that can cause serious medical effects on human health even at very low concentrations[2-4]. Therefore, routine detection of heavy metal ions is essential. Conventional analytical techniques for metal ion detection, including atomic absorption[5-7] and ICP-MS[8-10], are excellent in sensitivity and selectivity. However, they usually require expensive instruments, complicated sample preparation, or sophisticated operations[11]. Many efforts have been devoted to develop a highly sensitive, rapid, and selective analysis for heavy metals in the environment[12]. Unfortunately, most reported techniques are performed for the determination of individual metal ions in samples, which limits the throughput and efficiency. Simultaneous detection of heavy metal ions is increasingly required in aliment safety[13,14] and environmental monitoring[15,16]. Therefore, it is necessary to develop a simple, sensitive, and selective method for multiplex detection of heavy metal ions.

Much progress has been made with regard to multiplex assays for the detection of heavy metal ions[17-21]. Among them, microarrays have undoubtedly become an advanced research hotspot. Most microarrays are planar microarrays, in which the probe molecules are immobilized on a substrate and encoded by the coordinate of their positions[15,19,20]. More recently, suspension arrays, using self-encoded microcarriers as elements

Key Laboratory of Biomedical Functional Materials, School of Science, China Pharmaceutical University. Tongjia Xiang 24, Nanjing, Jiangsu, 210009, P.R. China. *Corresponding authors.

E-mail addresses:baofen_ye@163.com(B.Ye),shenweiyang@cpu.edu.cn(W.Shen). for multiplexing, have emerged as a successful biotechnological technique for multiplex and high-throughput assays[22,23]. Compared to traditional planar microarrays, suspension arrays have greater flexibility in preparation, rapid because of radial diffusion, good reproducibility, and high sensitivity[23]. A variety of encoding microcarriers have been applied to suspension arrays, including segmented nanorods[24-26], photopatterning particles[27], semiconductor quantum dots (QDs)[28], fluorescent particles[29], and photonic crystal (PhC) particles[30-32]. In particular, $\mathrm{PhC}$ particles, a well-known photonic nanomaterial with spatial order lattices that exhibit brilliant structural colors, show great advantages owing to their excellent optical properties, such as minimal spectral width, remarkable stability, and freedom from fluorescent background[33-36]. We have successfully employed the PhC particles as encoding microcarriers for multiplex detection [31]. Various types of $\mathrm{PhC}$ particles, such as close-packed $\mathrm{PhC}$ beads, non-close-packed $\mathrm{PhC}$ microparticles, and inverse opal beads, were developed for different analytes. Recently, multiplex metal ions detection was achieved using novel core-shell $\mathrm{PhC}$ microparticles generated by replicating and etching the silica close-packed $\mathrm{PhC}$ bead templates. The close-packed opal PhC cores provided stable diffraction peaks for encoding and the $\mathrm{PhC}$ hydrogel shells specifically recognized and reacted with the target heavy metal ions for sensing. As these core-shell $\mathrm{PhC}$ microparticles used ssDNA as the cross-linker to cause the shrinkage of the hydrogel shell, relatively high concentrations of ssDNA were required to observe the blue shift of the diffraction peak of the PhC shells. Using a high concentration of ssDNA not only increases the cost, but also reduces the detection sensitivity.

In contrast, fluorescent detection, which shows great advantages of high sensitivity, specificity, simplicity, and wide dynamic range in most suspension arrays, offers an excellent 
alternative. In this paper, we present a novel type of suspension array for multiplex detection of toxic metal ions with $\mathrm{PhC}$ hydrogel microspheres as encoding microcarriers, which use the hydrogel matrix for ssDNA immobilization and the ssDNA for fluorescent signal generation. The $\mathrm{PhC}$ hydrogel microsphere consists of two units: silica colloidal crystal beads (SCCBs) prepared by self-assembling of silica nanoparticles as encoding units and ssDNA-functionalized hydrogel as sensing units. When a typical target analysis process occurs, the ssDNA-functionalized hydrogel penetrated in the SCCBs could selectively recognize and bind with the target metal ions. The binding interaction between the ssDNA and its target could induce the conformation of ssDNA to a hairpin structure. Upon addition of SYBR Green I dye, green fluorescence will be observed (Scheme 1). When used in multiplex metal ions analyses, SCCBs could offer stable diffraction peaks for encoding information while the amount of the target metal ions can be quantitatively estimated by the fluorescence of the ssDNA-functionalized hydrogel. As hydrogels can provide a permeable 3-D soft scaffold, improve the binding kinetics, and reduce steric issues, our suspension array presents a successful detection of $\mathrm{Hg}^{2+}$ and $\mathrm{Ag}^{+}$in solution selectively and sensitively.

(A)

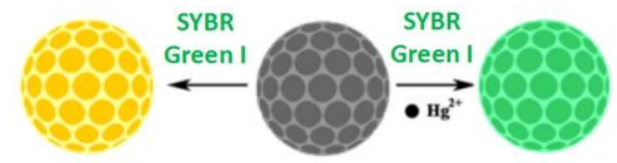

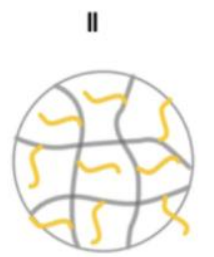

Yellow fluorescence
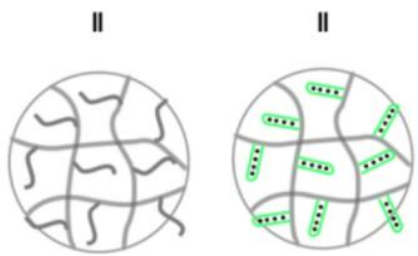

Green fluorescence
(B) 5' $\simeq: 5^{\prime}-\mathrm{NH}_{2}-\left(\mathrm{CH}_{2}\right)_{6}$-TTCTTTCTTCCCTTGTTTGTT-3'

Scheme 1 (A) ssDNA-functionalized PhC hydrogel microspheres and the interaction with $\mathrm{Hg}^{2+}$ and SYBR Green I. A weak orange fluorescence was observed with the addition of SYBR Green I. The addition of SYBR Green I and $\mathrm{Hg}^{2+}$ caused a hairpin configuration change of the ssDNA, resulting in a strong green fluorescence. (B) The ssDNA sequence used in this study and the fluorescence signal generation for $\mathrm{Hg}^{2+}$ detection. The 5'-end of SSDNA was modified with an amino group for hydrogel attachment.

\section{Material and Methods}

\section{Materials}

ssDNA probes (Table 1) were synthesized by Sangon Biotechnology Co., Ltd (Shanghai, China) and purified by standard desalting. SYBR Green I was purchased from Sangon Biotechnology Co., Ltd (Shanghai, China). Agarose and mercury (II) perchlorate were purchased from Alfa Aesar China Ltd. Sliver nitrate, epichlorohydrin, sodium bicarbonate, sodium hydroxide, sodium dihydrogen phosphate, and disodium hydrogen phosphate were obtained from Sinopharm Chemical Reagent Co., Ltd (Shanghai, China).

\section{Apparatus}

Photographs of the microspheres were obtained with an Olympus microscope (Olympus BX51). Reflection and fluorescence spectra of the microspheres were obtained at a fixed glancing angle by utilizing an optical microscope equipped with a fiber-optic spectrometer (Ocean Optics, USB2000-FLG). The microstructures of the beads were characterized by a scanning electron microscopy (SEM, Hitachi, S-300N). Fluorescence spectra were recorded on a RF-5301 spectrofluorimeter (Shimatdzu China Co., Ltd., Beijing, China). $\mathrm{pH}$ measurements were obtained using a pHS-25 $\mathrm{pH}$ meter (Shanghai Leici Chuangyi Apparatus \& Instrument Co., Ltd).

\section{Generation of SCCBS}

The SCCBs were fabricated by the droplet template method according to our previous work[31]. The aqueous $20 \mathrm{wt} \%$ silica nanoparticle suspension solution and oil were injected into the microfluidic device. The aqueous suspension was broken into droplets by the oil flows in the microfluidic channel. The droplets were collected into a container filled with hexeadecane oil containing $1 \%$ hypermer 2296 surfactant. The water in the droplets was evaporated at $80{ }^{\circ} \mathrm{C}$ overnight. After solidification, the SCCBs were calcined at $800{ }^{\circ} \mathrm{C}$ for $3 \mathrm{~h}$ to improve their mechanical strength.

\section{Fabrication of PhC hydrogel microspheres}

The fabrication of the PhC hydrogel microsphere was based on the SCCBs. The hydrogel used in this paper was $5 \%(\mathrm{v} / \mathrm{v})$ agarose solution. The agarose solution was heated at $80{ }^{\circ} \mathrm{C}$ until it became a transparent colorless solution. Then, the SCCBs were immersed in the continuously heating agarose solution for $30 \mathrm{~min}$. The SCCBs with the agarose solution were stored at room temperature until the hydrogel formed, as illustrated in 
Table 1

Sequences of ssDNA probes used in this work.

\begin{tabular}{cr}
\hline Name & Aptamer sequence \\
\hline $\mathbf{P}_{\mathrm{Hg}}$ & $5^{\prime}-\mathrm{NH} 2-\left(\mathrm{CH}_{2}\right)_{6}-$ TTCTTTCTTCCCTTGTTTGTT \\
\hline $\mathbf{P}_{\mathrm{Ag}}$ & 5'-NH2- $\left(\mathrm{CH}_{2}\right)_{6}-$ CTCTCTTCTCAAAAAACACAACACAC \\
\hline
\end{tabular}

Fig.1(A). The hydrogel was mechanically disrupted with hands gently until the hydrogel filled SCCBs, which we called photonic crystal $(\mathrm{PhC})$ hydrogel microspheres, could fall off from the hydrogel body. The obtained $\mathrm{PhC}$ hydrogel microspheres were put into the activation solution $(10 \%(\mathrm{v} / \mathrm{v})$ epichlorohydrin and $10 \%(\mathrm{v} / \mathrm{v}) \mathrm{NaOH}$ solution. After incubation at $40{ }^{\circ} \mathrm{C}$ for $15 \mathrm{~min}$, the $\mathrm{PhC}$ hydrogel microspheres were washed with pure water and $0.2 \mathrm{M} \mathrm{NaHCO}_{3}$ three times, respectively. Lastly, the activated $\mathrm{PhC}$ hydrogel microspheres were mixed with the corresponding ssDNA probe $(1.0 \mu \mathrm{M})$ in $0.2 \mathrm{M} \quad \mathrm{NaHCO}_{3}$ solution overnight and the ssDNA-functionalized $\mathrm{PhC}$ hydrogel microspheres were rinsed with pure water thoroughly for further use.

\section{Detection of metal ions}

The detection of metals ions was performed in Tris nitrate (pH 8.0, $20 \mathrm{mM}$ ) buffer. In a typical process, PhC hydrogel microspheres were first equilibrated in Tris nitrate buffer that contained 10000x SYBR Green I in a polypropylene tube (200 $\mu 1)$. Different concentrations of $\mathrm{Hg}^{2+}$ were added to the tube and incubated with the $\mathrm{PhC}$ hydrogel microspheres at room temperature for $30 \mathrm{~min}$ and fluorescence microscopy images were collected.

\section{Selectivity of the PhC hydrogel microspheres and multiplex detection}

The selectivity of $\mathrm{PhC}$ hydrogel microspheres for $\mathrm{Hg}^{2+}$ was evaluated by testing the response of the microspheres to other environmentally relevant metal ions, including $\mathrm{Ag}^{+}, \mathrm{Pb}^{2+}, \mathrm{Al}^{3+}$, $\mathrm{Ba}^{2+}, \mathrm{Zn}^{2+}$, and $\mathrm{Mg}^{2+}$, at a concentration of $1.0 \mu \mathrm{M}$. Multiplex detection was carried out by two kinds of $\mathrm{PhC}$ hydrogel microspheres functionalized by $\mathrm{P}_{\mathrm{Hg}}$ and $\mathrm{P}_{\mathrm{Ag}}$, respectively. These two kinds of microspheres were added into one test tube and incubated with a mixed solution containing $\mathrm{Hg}^{2+}(1.0 \mu \mathrm{M})$ and $\mathrm{Ag}^{+}(1.0 \mu \mathrm{M})$. After $30 \mathrm{~min}$ of incubation, the microspheres were washed with buffer five times. The fluorescence microscopy images were collected as described in the previous section.

\section{Detection of heavy metal ions in tap water samples}

To evaluate the applications of the proposed microspheres, tap water was collected locally. The sample was collected after discharging tap water for $30 \mathrm{~min}$ and boiling for $5 \mathrm{~min}$ to remove chlorine. The standard solutions of $\mathrm{Hg}^{2+}$ and $\mathrm{Ag}^{+}$were added to three tap water samples at three different concentrations $(0.01 \mu \mathrm{M}, 1 \mu \mathrm{M}$, and $10 \mu \mathrm{M})$ of each ion. Then, these water samples were analyzed using the proposed method.

\section{Results and Discussion}

(A)
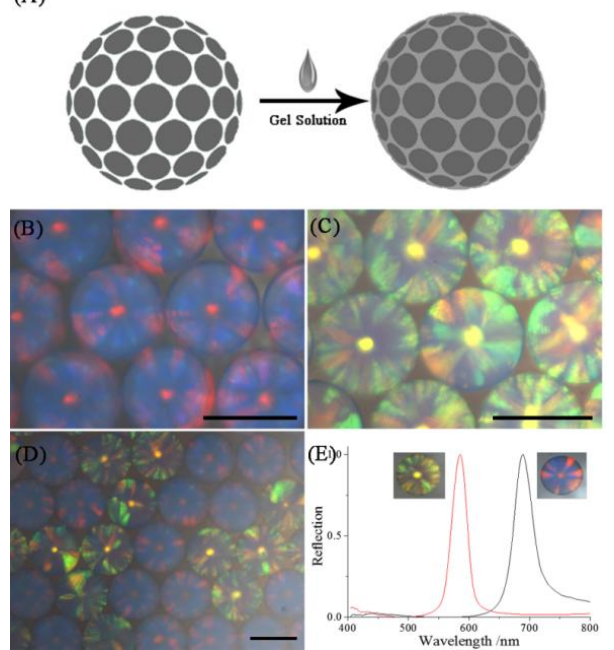

Fig. 1 (A) Schematic illustration of the fabrication of the $\mathrm{PhC}$ hydrogel microspheres. (B-D) The reflection images of two kinds of $\mathrm{PhC}$ hydrogel microspheres. (E) The reflection spectra of two kinds of $\mathrm{PhC}$ hydrogel microspheres. Scale bars are $200 \mu \mathrm{m}$.

The PhC hydrogel microspheres were composed of SCCB and the filled hydrogel throughout the nanopores of the SCCB. The hydrogel served as a substrate for ssDNA immobilization. Agarose was chosen as the hydrogel matrix owing to its non-toxicity, low cost, and simple formation. SCCBs with high monodispersity and brilliant structural colors were prepared by self-assembling of silica nanoparticles in microfluidic droplets. The structural colors of the SCCBs originated from the ordered arrangement of the spherical silica nanoparticles. These packed spherical nanoparticles formed connected nanopores throughout 
the SCCBs. The heated agarose solution entered these nanopores and filled all the empty spaces between the silica nanoparticles of the SCCBs by capillary force. After fully soaking, the mixture was cooled down to room temperature until the hydrogel formed. The hydrogel was then mechanically disrupted with hands and the SCCBs filled with agarose hydrogel (PhC hydrogel microspheres). A round smooth surface at the split could be obtained. We found that the hydrogel performance was influenced by the agarose percentage. High agarose concentration $(>10 \%)$ would generate stiff hydrogel while low agarose percentage $(<3 \%)$ would obtain too soft hydrogel, both of which are difficult to handle. Hence, a moderate agarose percentage (5\%) was chosen in this study. Under this condition, the $\mathrm{PhC}$ microspheres with uniform round surface and unique characteristic reflection peaks were prepared. Thanks to their unique characteristic reflection peaks, the $\mathrm{PhC}$ microspheres were employed as encoded microcarriers for the suspension assay. According to Bragg's law $(\lambda=2 n d \sin \theta$, where $\lambda$ is the diffraction wavelength, $d$ is the lattice plane spacing, $n$ is the average refractive index and $\theta$ is the Bragg angle), the reflection peak position can be controlled by the diameter of silica nanoparticles during the SCCB fabrication. Therefore, two PhC hydrogel microspheres with the same size (Fig. 1(B-D)) but different diffraction-peak positions were obtained (Fig. 1(E)).

For ssDNA immobilization, the $\mathrm{PhC}$ microspheres were first activated by epichlorohydrin. As ssDNA was immobilized for fluorescent signal generation, the ssDNA concentration used was $1.0 \mu \mathrm{M}$ to observe the fluorescence change of SYBR Green I dye in the presence of heavy metal ions. Detection was achieved by selective binding of heavy metal ions with the probe ssDNA, which induced the ssDNA to form a dsDNA hairpin structure. SYBR Green I could then intercalate into dsDNA and generate a strong fluorescence signal. In order to verify the feasibility of the proposed strategy, an experiment for proof of principle was performed. As shown in Fig. 2, in the absence of $\mathrm{Hg}^{2+}$, the ssDNA adopted a random coil structure and SYBR Green I could interact with the added ssDNA through electrostatic interactions and increase the fluorescence of SYBR Green I slightly (curve b)[37]. Upon the addition of $\mathrm{Hg}^{2+}$, the aptamer formed a hairpin structure. Once $\mathrm{Hg}^{2+}$ was added into the system, the ssDNA formed a hairpin structure. SYBR Green I could bind to dsDNA through intercalation and minor groove binding, resulting in a great enhancement of fluorescence (curve $\mathrm{c}$ and d). When ssDNA or no DNA existed in the sensing system, a weak fluorescence signal was observed (curve $a$ and $b$ ). This demonstrated that SYBR Green I bound strongly to the dsDNA over ssDNA as expected.

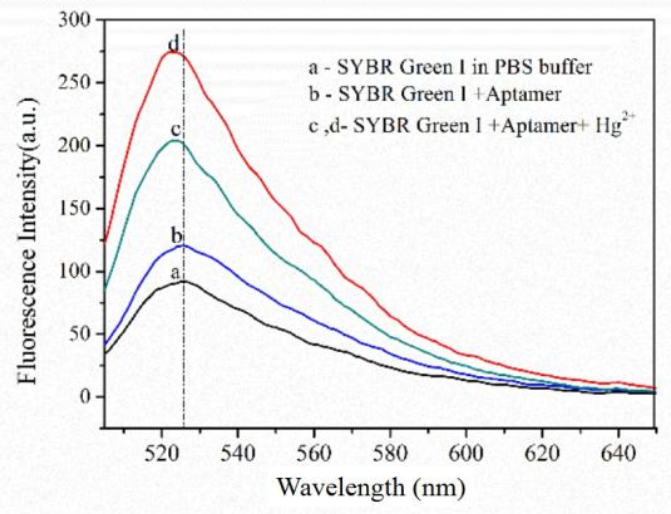

Fig. 2 (a) Fluorescence spectra of SYBR Green I (1000X) solution. (b) Fluorescence spectra of SYBR Green I (1000X) solution contained 1.0 $\mu \mathrm{M} \mathrm{P}_{\mathrm{Hg}}$. (c) Fluorescence spectra of SYBR Green I (1000X) solution contained $1.0 \mu \mathrm{M} \mathrm{P} \mathrm{P}_{\mathrm{Hg}}$ and $2.0 \mu \mathrm{M} \mathrm{Hg}^{2+}$. (d) Fluorescence spectra of SYBR Green I (1000X) solution contained $1.0 \mu \mathrm{M} \mathrm{P}_{\mathrm{Hg}}$ and $4.0 \mu \mathrm{M}$ $\mathrm{Hg}^{2+}$.

To investigate the activation effect of epichlorohydrin, we used the detection of single-analyte $\mathrm{Hg}^{2+}$. A series of control experiments was carried out to evaluate and optimize the detection conditions. First, to confirm whether the heavy metal ion-induced fluorescence enhancement was caused by the selective binding of ions with probe ssDNA, $\mathrm{PhC}$ hydrogel microspheres immobilized with and without probe $\operatorname{ssDNA}\left(\mathrm{P}_{\mathrm{Hg}}\right)$ were tested. Almost no fluorescence could be observed after the addition of SYBR Green I (Fig. 3(A)). This control experiment suggests that $\mathrm{Hg}^{2+}$-induced fluorescence enhancement is indeed caused by the specific interaction of $\mathrm{Hg}^{2+}$ with $\mathrm{P}_{\mathrm{Hg}}$. The control experiment with non-activated $\mathrm{PhC}$ hydrogel microspheres (as shown in Fig 3(B)) proved that only after the activation procedure can the probe ssDNA be immobilized on the $\mathrm{PhC}$ hydrogel microspheres. Different activation time also caused a variety of fluorescence responses. Usually, a long activation time is expected to yield an enhanced signal, while, for practical use, a shorter assay time is preferred. Therefore, the activation time was experimentally optimized. As shown in Fig. 3(C), fluorescence intensity increased with the activation time at short activation times. After activation for $15 \mathrm{~min}$, the fluorescence reached a maximum. Further activation time did not lead to an obvious fluorescence enhancement. Thus, an activation time of 15 min was chosen for subsequent experiments. 
(A)

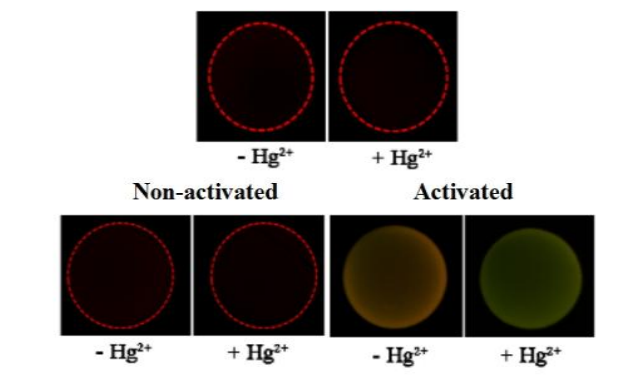

(C)

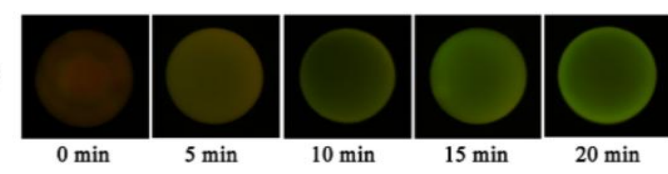

Fig. 3 (A) Control experiments with $\mathrm{PhC}$ hydrogel microspheres containing no ssDNA. (B) Control experiments with non-activated $\mathrm{PhC}$ hydrogel microspheres (left) and activated $\mathrm{PhC}$ hydrogel microspheres (right). (C) $\mathrm{PhC}$ hydrogel microspheres fluorescence changes as a function of activation time.

To test the sensitivity of our PhC hydrogel microspheres, the $\mathrm{P}_{\mathrm{Hg}}$ immobilized $\mathrm{PhC}$ hydrogel microspheres were soaked in different concentrations of $\mathrm{Hg}^{2+}$. As shown in Fig. 4(A), the fluorescence of the microspheres showed a remarkable change in the presence of $\mathrm{Hg}^{2+}$. When the concentration of $\mathrm{Hg}^{2+}$ solution increased, the fluorescence enhancement was more apparent. The plot of fluorescence intensity of the $\mathrm{PhC}$ hydrogel microsphere versus $\mathrm{Hg}^{2+}$ concentration was shown in Fig. 4(B). The detection limit was determined to be $1 \mathrm{nM}$ under the signal-to-noise ratio of $3: 1$. The selectivity was also demonstrated by testing the response of the $\mathrm{P}_{\mathrm{Hg}}$ immobilized $\mathrm{PhC}$ hydrogel microspheres to other environmentally relevant metal ions. It was observed that only $\mathrm{Hg}^{2+}$ could induce a significant fluorescence enhancement for the microspheres (Fig. 4(C)). The microspheres displayed an undetectable fluorescence intensity response to the control metal ions. In addition, $1 \mu \mathrm{M}$ $\mathrm{Hg}^{2+}$ in the presence of 50-fold interfering $\mathrm{Pb}^{2+}$ and 50 -fold interfering $\mathrm{Ag}^{+}$was detected. No significant fluorescence change was found in comparison with that in the absence of $\mathrm{Pb}^{2+}$ and $\mathrm{Ag}^{+}$. Thus, our PhC hydrogel microspheres showed good selectivity against other relevant metal ions.
(A)

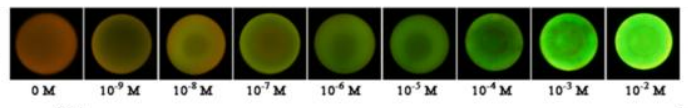

(B)

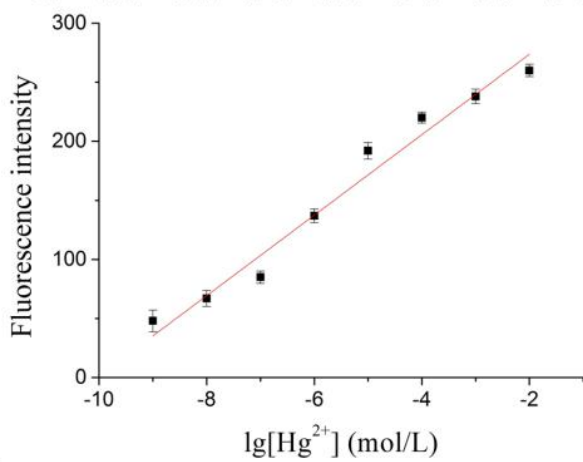

(C)

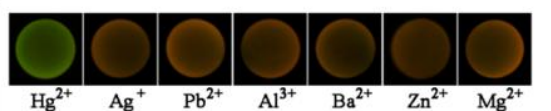

Fig. 4 (A) Sensitivity of the $\mathrm{PhC}$ hydrogel microsphere detected using a fluorescence microscope. (B) Plot of fluorescence intensity of the PhC hydrogel microsphere with different $\mathrm{Hg}^{2+}$ concentrations. Error bars represent standard deviation of five experiments. (C) Selectivity test with $1.0 \mu \mathrm{M}$ concentrations of various metal ions.

To demonstrate the reliability of $\mathrm{PhC}$ hydrogel microspheres in sensing multiple types of metal ions, two types of $\mathrm{PhC}$ hydrogel microspheres with the characteristic reflection peaks at $585 \mathrm{~nm}$ and $690 \mathrm{~nm}$ that exhibit green and red structural colors were immobilized with two kinds of ssDNA probes, $\mathrm{P}_{\mathrm{Hg}}$ and $\mathrm{P}_{\mathrm{Ag}}$, respectively (Fig. 5(A)). These ssDNA-immobilized $\mathrm{PhC}$ hydrogel microcapsules were then mixed and incubated in a multi-target solution of $\mathrm{Hg}^{2+}$ and $\mathrm{Ag}^{+}$. Because of the specific binding between the probe ssDNA and their corresponding ions, the fluorescence intensity of the microspheres showed significant enhancement only in the presence of their corresponding target ions, and none of the microspheres showed significant fluorescence enhancement in response to non-target ions. In the absence of the target metal ions, no fluorescence changes could be observed from both microspheres (Fig. 5(B)). Fig. 5(C) represented typical fluorescence images of the microspheres when they were incubated with a single target $\mathrm{Hg}^{2+}(1.0 \mu \mathrm{M})$. The $\mathrm{P}_{\mathrm{Hg}}$ immobilized microsphere presented an intense green fluorescence, while the $\mathrm{P}_{\mathrm{Ag}}$-immobilized microsphere presented no fluorescence changes. Fig. 5(D) showed the result of two kinds of $\mathrm{PhC}$ hydrogel microspheres in the presence of $\mathrm{Hg}^{2+}(1.0 \mu \mathrm{M})$ and $\mathrm{Ag}^{+}(1.0 \mu \mathrm{M})$ multi-target solution. Both of the microspheres yielded a significant fluorescence enhancement to its corresponding target ions. All of the above results indicated that the $\mathrm{PhC}$ hydrogel microspheres could be successfully applied to multiplex heavy 
metal ion detection.
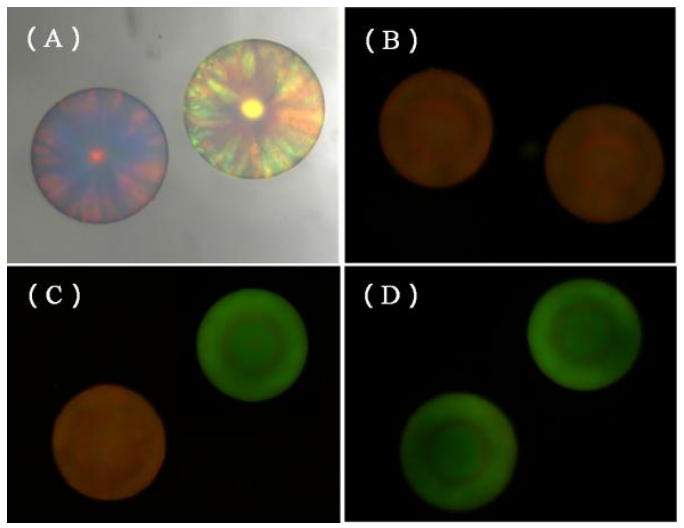

Fig. 5 (A) The reflection image of two kinds of $\mathrm{PhC}$ hydrogel microspheres. (B) The fluorescence image of two kinds of PhC hydrogel microspheres in the absence of target metal ions. (C) The fluorescence image of two kinds of PhC hydrogel microspheres in the presence of target $\mathrm{Hg}^{2+}$. (D) The fluorescence image of two kinds of PhC hydrogel microspheres in the presence of target $\mathrm{Hg}^{2+}$ and $\mathrm{Ag}^{+}$. recognized and reacted with target ions. Upon treatment with the SYBR Green I dye, the fluorescence intensity was changed and used to estimate the amounts of target metal ions. We anticipate that the microcarriers would provide a promising platform for multiplex applications.

\section{Acknowledgements}

This work was supported by the National Science Foundation of China (Grant No. 51403237), the National Science Foundation of Jiangsu (Grant No. BK20140658), the Open Research Fund of State Key Laboratory of Bioelectronics, Southeast University. B.F.Y. also thanks the Postdoctoral Science Foundation of China and Jiangsu province (Grant Nos. 2014M561544 and 1401036B).

\section{Table 2}

Determination of heavy metal ions in tap water samples using the proposed microspheres.

\begin{tabular}{lccccccc}
\hline \multicolumn{2}{l}{ Spiked amount $(\mu \mathrm{M})$} & \multicolumn{2}{c}{ Measured amount $(\mu \mathrm{M})$} & \multicolumn{2}{c}{ Recovery $(\%)$} & \multicolumn{2}{c}{$\mathrm{RSD}(\%, n=3)$} \\
$\mathrm{Hg}^{2+}$ & $\mathrm{Ag}^{+}$ & $\mathrm{Hg}^{2+}$ & $\mathrm{Ag}^{+}$ & $\mathrm{Hg}^{2+}$ & $\mathrm{Ag}^{+}$ & $\mathrm{Hg}^{2+}$ & $\mathrm{Ag}^{+}$ \\
\hline 0.01 & 0.01 & 0.011 & 0.010 & 110 & 100 & 8.7 & 7.0 \\
\hline 1.0 & 1.0 & 0.93 & 1.05 & 93.0 & 105 & 5.6 & 6.8 \\
\hline 10.0 & 10.0 & 9.5 & 9.7 & 95.0 & 97.0 & 4.9 & 5.3 \\
\hline
\end{tabular}

The ability of our microspheres to detect heavy metal ions in tap water samples was then evaluated. The sample was collected after discharging tap water for $30 \mathrm{~min}$ and boiling for $5 \mathrm{~min}$ to remove chlorine. Three spiked samples at three different concentrations $(0.01$ $\mu \mathrm{M}, 1 \mu \mathrm{M}$, and $10 \mu \mathrm{M}$ ) of each ion were prepared by adding the standard solutions of $\mathrm{Hg}^{2+}$ and $\mathrm{Ag}^{+}$into the tap water. As shown in Table 2, good average recovery rates from $93.0 \%$ to $110 \%$ for $\mathrm{Hg}^{2+}$ detection and $97.0 \%$ to $105 \%$ for $\mathrm{Ag}^{+}$detection were obtained, which suggests that our method was largely free from the matrix effect of real water samples.

\section{Conclusions}

In summary, we developed an excellent suspension array for multiplex detection of heavy metal ions with $\mathrm{PhC}$ hydrogel microspheres as encoding microcarriers and ssDNA probes as sensing units. The $\mathrm{PhC}$ microspheres provided stable diffraction peaks for encoding and the ssDNA-functionalized hydrogel

\section{References}

[1] J. Wang, C. Chen, Biosorption of heavy metals by Saccharomyces cerevisiae: a review, Biotechnology Advances, 24 (2006) 427-451.

[2] H.H. Harris, I.J. Pickering, G.N. George, The Chemical Form of Mercury in Fish, Science, 301 (2003) 1203.

[3] T. Kang, S.M. Yoo, M. Kang, H. Lee, H. Kim, S.Y. Lee, B. Kim, Single-step multiplex detection of toxic metal ions by $\mathrm{Au}$ nanowires-on-chip sensor using reporter elimination, Lab on A Chip, 12 (2012) 3077-3081.

[4] C. Zhou, W. Jiang, B.K. Via, Facile synthesis of soluble graphene quantum dots and its improved property in detecting heavy metal ions, Colloids \& Surfaces B Biointerfaces, 118 (2014) 72-76.

[5] C. He, G. Cheng, C. Zheng, L. Wu, Y.I. Lee, X. Hou, Photochemical vapor generation and in situ 
preconcentration for determination of mercury by graphite furnace atomic absorption spectrometry, Analytical Methods, 7 (2015) 3015-3021.

[6] P. Padmaja, N. Balasubramanian, T.V. Ramakrishna, Determination of Submicrogram Amounts of Inorganic and Organo-Mercury Compounds by Absorption Spectrophotometry, International Journal of Environmental Analytical Chemistry, 63 (1996) 47-59.

[7] J. Pandey, P. Sudhakar, V.J. Koshy, Determination of silver at submicrogram levels by absorption spectrophotometry, Indian Journal of Chemical Technology, 10 (2003) 295-297.

[8] R.S. Picoloto, H. Wiltsche, G. Knapp, J.S. Barin, E.M.M. Flores, Mercury determination in soil by CVG-ICP-MS after volatilization using microwave-induced combustion, Analytical Methods, 4 (2011) 630-636.

[9] S. Slaets, F. Adams, I.R. Pereiro, R. Łobiński, Optimization of the coupling of multicapillary GC with ICP-MS for mercury speciation analysis in biological materials, Journal of Analytical Atomic Spectrometry, 14 (1999) 851-857.

[10] D. Li, Z. Zhao, Q. Chu, J. Fang, Sulfhydryl Cotton Enrichment Separation-Determination of Silver in Geological Samples by ICP-MS, Bulletin- Korean Chemical Society, 32 (2011) 3561-3565.

[11] M. Leermakers, W. Baeyens, P. Quevauviller, M. Horvat, Mercury in environmental samples: Speciation, artifacts and validation, Trac Trends in Analytical Chemistry, 24 (2005) 383-393.

[12] L. Feng, A. Zhu, H. Shi, H. Wang, J. Liu, Rapid on-site/in-situ detection of heavy metal ions in environmental water using a structure-switching DNA optical biosensor, Scientific Reports, 3 (2013) 2308.

[13] C.Y. Cho, W. Nowatzke, K. Oliver, E.A.E. Garber, Multiplex detection of food allergens and gluten, Analytical and Bioanalytical Chemistry, 407 (2015) 4195-4206.

[14] C. Unterberger, F. Luber, A. Demmel, K. Grünwald, I. Huber, K.H. Engel, U. Busch, Simultaneous detection of allergenic fish, cephalopods and shellfish in food by multiplex ligation-dependent probe amplification, European Food Research and Technology, 239 (2014) 559-566.

[15] Y. Huang, F. Li, M. Qin, L. Jiang, Y. Song, A multi-stopband photonic-crystal microchip for high-performance metal-ion recognition based on fluorescent detection, Angewandte Chemie International Edition, 125 (2013) 7296-7299.

[16] Y. Yang, S.K. Seidlits, M.M. Adams, V.M. Lynch, C.E. Schmidt, E.V. Anslyn, J.B. Shear, A highly selective low-background fluorescent imaging agent for nitric oxide, Journal of the American Chemical Society, 132 (2010) 13114-13116.

[17] L.Y. Niu, H. Li, L. Feng, Y.S. Guan, Y.Z. Chen, C.F. Duan, L.Z. Wu, Y.F. Guan, C.H. Tung, Q.Z. Yang, BODIPY-based fluorometric sensor array for the highly sensitive identification of heavy-metal ions, Analytica Chimica Acta, 775 (2013) 93-99.

[18] M. Bally, M. Graule, F. Parra, A virus biosensor with single virus-particle sensitivity based on fluorescent vesicle labels and equilibrium fluctuation analysis, Biointerphases, 8 (2013) 4.

[19] Q. Zhao, S. Chen, L. Zhang, H. Huang, Y. Zeng, F. Liu, Multiplex sensor for detection of different metal ions based on on-off of fluorescent gold nanoclusters, Analytica Chimica Acta, 852 (2014) 236-243.

[20] Y. Huang, F. Li, C. Ye, M. Qin, W. Ran, Y. Song, A photochromic sensor microchip for high-performance multiplex metal ions detection, Scientific Reports, 5 (2015) 9724.

[21] J. Xia, M. Lin, X. Zuo, S. Su, L. Wang, W. Huang, C. Fan, Q. Huang, Metal ion-mediated assembly of DNA nanostructures for cascade fluorescence resonance energy transfer-based fingerprint analysis, Analytical Chemistry, 86 (2014) 7084-7087.

[22] Dayang Wang, Andrey L. Rogach, Frank CarusoMax Planck, Semiconductor Quantum Dot-Labeled Microsphere Bioconjugates Prepared by Stepwise Self-Assembly, Nano Letters, 2 (2002) 857-861.

[23] B. He, S.J. Son, S.B. Lee, Suspension array with shape-coded silica nanotubes for multiplexed immunoassays, Analytical Chemistry, 79 (2007) 5257-5263.

[24] R.W. Dr, A.R.C. Prof, D.G.S. Dr, Encoded Microcarriers 
For High-Throughput Multiplexed Detection, Angewandte Chemie International Edition, 45 (2006) 6104-6117.

[25] S. Li, L. Xu, W. Ma, H. Kuang, L. Wang, C. Xu, Triple Raman Label-Encoded Gold Nanoparticle Trimers for Simultaneous Heavy Metal Ion Detection, Small, 11 (2015) 3435-3439.

[26] J. Zhang, L. Wang, H. Zhang, F. Boey, S. Song, C. Fan, Aptamer-based multicolor fluorescent gold nanoprobes for multiplex detection in homogeneous solution, Small (Weinheim an der Bergstrasse, Germany), 6 (2010) 201-204.

[27] W. Lee, D. Choi, J.H. Kim, W.G. Koh, Suspension arrays of hydrogel microparticles prepared by photopatterning for multiplexed protein-based bioassays, Biomedical Microdevices, 10 (2008) 813.

[28] Y. Zhao, H.C. Shum, H. Chen, L.L.A. Adams, Z. Gu, D.A. Weitz, Microfluidic Generation of Multifunctional Quantum Dot Barcode Particles, Journal of the American Chemical Society, 133 (2011) 8790-8793.

[29] Bronwyn J. Battersby, Darryn Bryant, Wim Meutermans, Daniel Matthews, a. Mark L. Smythe, Matt Trau, Toward Larger Chemical Libraries: Encoding with Fluorescent Colloids in Combinatorial Chemistry, Journal of the American Chemical Society, 122 (2000) 2138-2139.

[30] Y. Zhao, Y. Cheng, L. Shang, J. Wang, Z. Xie, Z. Gu, Microfluidic synthesis of barcode particles for multiplex assays, Small, 11 (2014) 151-174.

[31] B. Ye, H. Ding, Y. Cheng, H. Gu, Y. Zhao, Z. Xie, Z. Gu, Photonic crystal microcapsules for label-free multiplex detection, Advanced Materials, 26 (2014) 3270-3274.

[32] F. Cunin, T.A. Schmedake, J.R. Link, Y.Y. Li, J. Koh, S.N. Bhatia, M.J. Sailor, Biomolecular screening with encoded porous-silicon photonic crystals, Nature Materials, 1 (2002) 39.

[33] Y. Zhao, Z. Xie, H. Gu, C. Zhu, Z. Gu, Bio-inspired variable structural color materials, Chemical Society Reviews, 41 (2012) 3297.

[34] D. Wang, J. Li, C.T. Chan, L.M. Liz - Marzán, S. Romanov, F. Caruso, Optical Properties of Nanoparticle - Based Metallodielectric Inverse
Opals, Small, 1 (2005) 122.

[35] M. Qin, Y. Huang, Y. Li, M. Su, B. Chen, H. Sun, P. Yong, C. Ye, F. Li, Y. Song, A Rainbow Structural Color Chip for Multisaccharide Recognition, Angewandte Chemie, 128 (2016) 7025-7028.

[36] Y. Huang, J. Zhou, B. Su, L. Shi, J. Wang, S. Chen, L. Wang, J. Zi, Y. Song, L. Jiang, Colloidal photonic crystals with narrow stopbands assembled from low-adhesive superhydrophobic substrates, Journal of the American Chemical Society, 134 (2012) 17053.

[37] L.Q. Guo, N. Yin, D.D. Nie, J.R. Gan, M.J. Li, F.F. Fu, G.N. Chen, Label-free fluorescent sensor for mercury(II) ion by using carbon nanotubes to reduce background signal, The Analyst, 136 (2011) 1632-1636. 


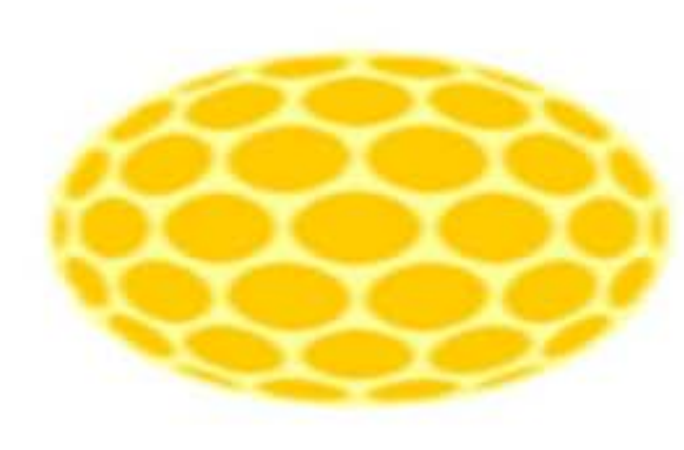

II

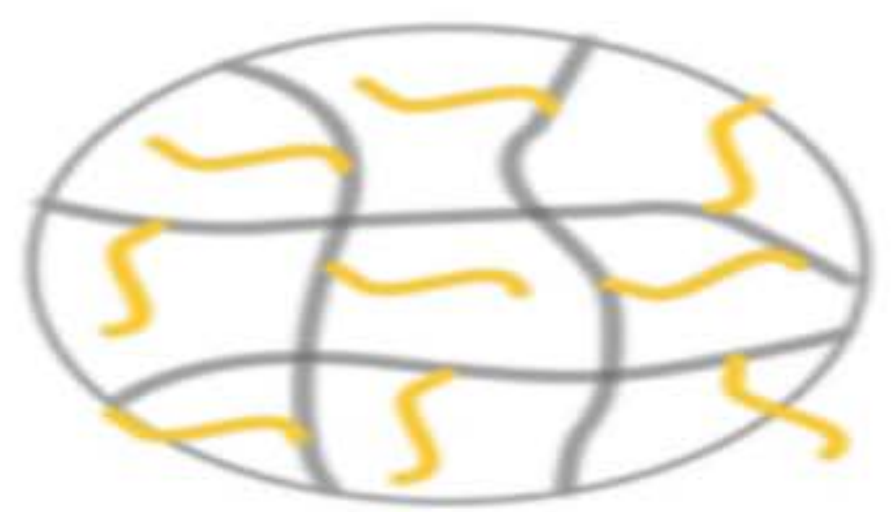

Yellow fluorscence

\section{SYBR}

Green I

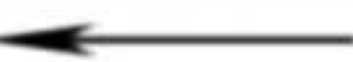

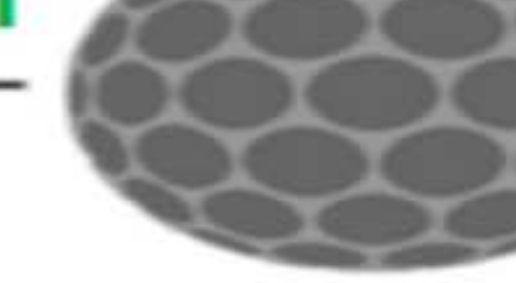

II

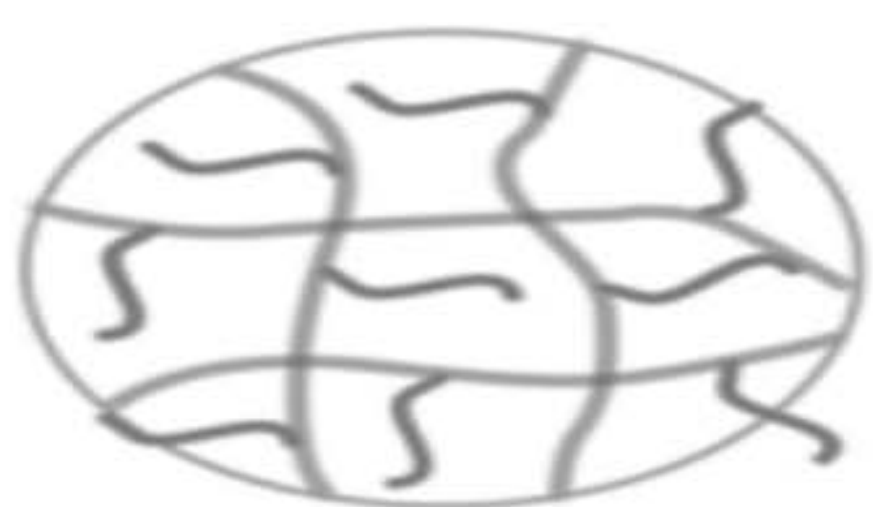

SYBR

Green I

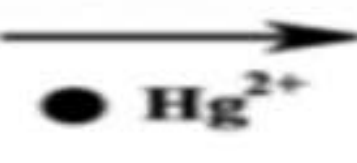

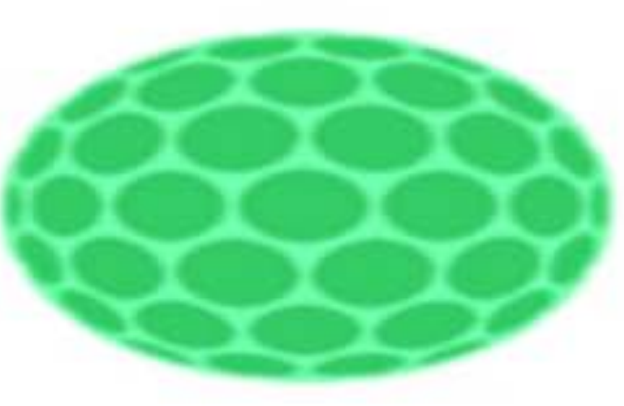

II

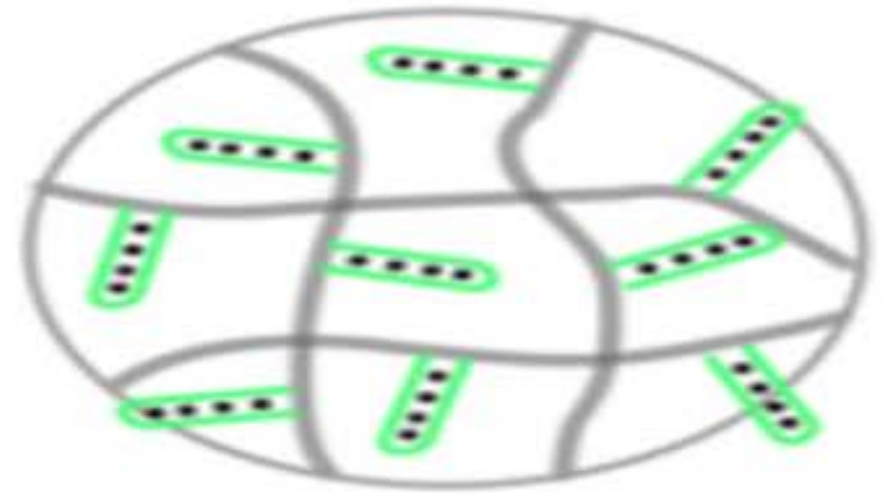

Green fluorscence 